TITLE:

\title{
Personal reminiscence: combinatorial and discrete optimization problems in which I have been interested
}

\author{
$\operatorname{AUTHOR}(\mathrm{S}):$ \\ Fujishige, Satoru
}

\section{CITATION:}

Fujishige, Satoru. Personal reminiscence: combinatorial and discrete optimization problems in which I have been interested. Japan Journal of Industrial and Applied Mathematics 2012, 29(3): 357-384

ISSUE DATE:

2012-10

URL:

http://hdl.handle.net/2433/167740

\section{RIGHT:}

The final publication is available at www.springerlink.com; This is not the published version. Please cite only the published version.; この論文 は出版社版でありません。引用の際には出版社版をご確認ご利用くだ さい。 


\title{
Personal Reminiscence-Combinatorial and Discrete Optimization Problems in Which I Have Been Interested
}

\author{
Satoru FUJISHIGE
}

Received: date / Accepted: date

\begin{abstract}
The present article takes up some of the author's research activities in the field of combinatorial and discrete optimization from 1975 till quite recently. It treats not only what the author has done but also what he still wants to do. They are written together with personal reminiscence and with the hope that this article will convey to researchers of younger generation the author's enthusiasm in combinatorial and discrete optimization on the frontier of research in the past till recently.
\end{abstract}

Keywords Combinatorial optimization · Discrete optimization

\section{Introduction}

My study (research) on combinatorial and discrete optimization started when I became a Research Associate at Professor Masao Iri's laboratory at the University of Tokyo in April, 1975 after I finished my Ph.D. study at Kyoto University. During my doctoral study under the guidance of Professors Yoshikazu Sawaragi and Tohru Katayama I made research in the field of estimation and control of stochastic dynamical systems. So I found very fresh and interesting the talks on discrete optimization and algorithms given at the seminars of Professor Iri's laboratory. My research in the field of combinatorial and discrete optimization really started in the summer of 1975 . The present article takes up some of my research activities in the field of combinatorial and discrete optimization from 1975 till quite recently, with my very personal reminiscence.

Throughout the present article we give rough ideas without precise definitions, so that the monograph [45] or relevant papers should be referred to for more details.

Research Institute for Mathematical Sciences, Kyoto University, Kyoto 606-8502, Japan

E-mail: fujishig@kurims.kyoto-u.ac.jp 


\section{Initial Work: Combinatorial controllability and observability}

I learned from Iri in the summer of 1975 that it was left open to solve the intersection problem on the two special infinite matroids arising from the controllability and observability of dynamical linear systems. The problem formulation on two infinite matroids was made by Iri and Nobuaki Tomizawa. Since I was familiar with linear control systems, I felt their problem close to me and started examining it.

It is the problem of discerning whether a given dynamical linear discrete-time system

$$
x(t+1)=A x(t)+B u(t), \quad(t=0,1, \cdots)
$$

is controllable under certain combinatorial constraints. Here $A$ and $B$ are, respectively, $n \times n$ and $n \times m$ real matrices, $x(t)$ is the $n$-dimensional column state vector at time $t$, and $u(t)$ is the $m$-dimensional column input vector at time $t$. We assume that the input terminals (corresponding to $m$ components of input vector $u(t)$ ) have physical meanings. Then the problem is

(C) to discern whether for any specified initial state and any final state there exists a finite sequence of input vectors that brings the initial state to the final state under combinatorial constraints:

(C1) at every time instance $t$ we can use at most $p$ input terminals ( $p$ : a fixed positive integer less than $m$ ) or

(C2) we can use each input terminal $i$ at most $q_{i}$ times $\left(q_{i}\right.$ : a fixed positive integer for $i=1, \cdots, m)$.

The observability problem with combinatorial constraints on output terminals is also similarly formulated.

It is well-known that without such combinatorial constraints the controllability (C) is equivalent to that the rank of the matrix

$$
\left[B|A B| \cdots \mid A^{n-1} B\right]
$$

is equal to $n$, and that we need only at most $n$ inputs $u(t)$ for $t=0,1, \cdots, n-1$.

Iri and Tomizawa formulated the above problem (C) with combinatorial constraints $(\mathrm{C} 1)$ or $(\mathrm{C} 2)$ as the intersection problem for two infinite matroids: one is given by the linear matroid on the column set of the infinite matrix

$$
\left[B|A B| \cdots\left|A^{n-1} B\right| \cdots \cdots\right]
$$

and the other given by

(C1) the direct sum of the uniform matroids $\mathbf{M}_{t}$ on the common input terminal set of rank $p$ for $t=0,1, \cdots$ or

(C2) the direct sum of the uniform matroids $\mathbf{M}_{i}$ on the infinite set of time instances $t=0,1, \cdots$ of rank $q_{i}$ for input terminals $i=1, \cdots, m$.

I showed that for both $(\mathrm{C} 1)$ and $(\mathrm{C} 2)$ the maximum cardinality of a common independent set of the two infinite matroids is attained by a common independent set on the two matroids restricted on the finite time horizon up to $t=n-1$ as is the case 
for the classical controllability without any constraints. My proof for (C1) was based on the Jordan canonical form of matrix $A$, and Iri gave an elegant proof by using the minimal polynomial for $A$, which lead us to the joint paper [77].

This joint work with Iri and Tomizawa further motivated me to shift my research to matroid optimization problems.

\section{Matroids, Polymatroids, and Submodular Optimization}

3.1 The independent assignment problem

In 1974 Iri and Tomizawa, and Eugine Lawler independently devised primal-dual polynomial algorithms for the independent assignment problem and equivalently the weighted matroid intersection problem (see [76] and [84]).

Early in 1976 I adapted Klein's primal min-cost flow algorithm to the independent assignment problem to get a new primal algorithm for it ([26]). Iri suggested me that the independent assignment problem could be extended in such a way that the bipartite matching problem was extended to Menger's (directed) arc-disjoint path problem. This resulted in [27], where the extended notion of arc-disjoint paths is called an independent linkage that links, through arc-disjoint paths, the independent set of entrances and that of exits when matroidal structures are given on the entrance set and the exit set (also see [74]).

Iri also told me that he did not know how the matroid optimization problem such as the independent assignment problem and the weighted matroid intersection problem could naturally be extended to a combinatorial optimization problem of flow type.

\subsection{The independent flow problem}

It was in the summer of 1976 that I found a paper of Colin McDiarmid [91] at the library of the department where Iri and I were working. Through his paper [91] and Jack Edmonds' [17] I learned the polymatroid theory and found a way of extending matroidal problems to flow problems with polymatroidal constraints. Then I reached a combinatorial optimization model, with polymatroids, of maximum flow and mincost flow type, which I called the independent-flow problem [28]. This naturally generalized the independent-linkage problem into flows in a network with an entrance set and an exit set, each being endowed with a polymatroidal structure. I gave a series of talks at Iri's laboratory in the fall of 1976 and presented the result at a meeting of the Technical Group on Circuits and Systems of the Institute of Electronics and Communication Engineers of Japan in December, 1976 and also at the Annual Spring Conference of the Operations Research Society of Japan in April, 1977.

In order to construct an algorithm for solving the independent-flow problem we needed some new concepts for polymatroids. For a polymatroid $(V, \rho)$ on a set $V$ 
with a rank function $\rho$ on $2^{V} \rightarrow \mathbf{Z}_{+}$(the set of nonnegative integers) the polymatroid polytope (or independence polytope) is given by

$$
\mathrm{P}_{(+)}(\rho)=\left\{x \in \mathbf{R}^{V} \mid \forall X \subseteq V: x(X) \leq \rho(X), x \geq \mathbf{0}\right\},
$$

where $x(X)=\sum_{v \in X} x(v)$ for all $X \subseteq V$, and also the base polytope by

$$
\mathrm{B}(\rho)=\left\{x \mid x \in \mathrm{P}_{(+)}(\rho), x(V)=\rho(V)\right\} .
$$

When $x(X)=\rho(X)$ for $x \in \mathrm{P}_{(+)}(\rho)$ and $X \subseteq V$, we say $X$ is tight for $x$.

Given an independent vector $x \in \mathrm{P}_{(+)}(\rho)$, an element $v \in V$ is said to be saturated if we cannot increase $x(v)$ without leaving $\mathrm{P}_{(+)}(\rho)$, i.e., $x+\alpha \chi_{v} \notin \mathrm{P}_{(+)}(\rho)$ for all $\alpha>0$. The set of saturated elements is denoted by $\operatorname{sat}(x)$. The set $\mathcal{F}(x)$ of all tight sets for $x$ is closed with respect to set union and intersection and $\operatorname{sat}(x)$ is the maximum element of $\mathcal{F}(x)$. For any element $v \in V$ we define the saturation capacity $\hat{\mathrm{c}}(x, v)$ by

$$
\hat{\mathrm{c}}(x, v)=\max \left\{\alpha \geq 0 \mid x+\alpha \chi_{v} \in \mathrm{P}_{(+)}(\rho)\right\} .
$$

We have $\hat{\mathrm{c}}(x, v)=0$ if and only if $v \in \operatorname{sat}(x)$.

Moreover, for any $x \in \mathrm{P}_{(+)}(\rho)$ and $u \in \operatorname{sat}(x)$ we define the dependence set associated with $x$ and $u$ by

$$
\operatorname{dep}(x, u)=\left\{v \in V \mid \exists \alpha>0: x+\alpha\left(\chi_{u}-\chi_{v}\right) \in \mathrm{P}_{(+)}(\rho)\right\},
$$

which is characterized as the minimum tight set that contains $u$. For any $v \in \operatorname{dep}(x, u)$ with $v \neq u$ we define the exchange capacity $\tilde{\mathrm{c}}(x, u, v)$ by

$$
\tilde{\mathrm{c}}(x, u, v)=\max \left\{\alpha \geq 0 \mid x+\alpha\left(\chi_{u}-\chi_{v}\right) \in \mathrm{P}_{(+)}(\rho)\right\} \quad(>0) .
$$

In [28] primal and primal-dual algorithms are proposed by using the saturation capacity and the exchange capacity given as oracles. It should be noted that equations (4) and (6) are also expressed as

$$
\begin{gathered}
\hat{\mathrm{c}}(x, v)=\min \{\rho(X)-x(X) \mid v \in X \subseteq V\}, \\
\tilde{\mathrm{c}}(x, u, v)=\min \{\rho(X)-x(X) \mid u \in X \subseteq V, v \notin X\} .
\end{gathered}
$$

Hence, the problem of computing $\hat{\mathrm{c}}(x, v)$ and $\tilde{\mathrm{c}}(x, u, v)$ is submodular function minimization.

In most practical applications we may compute the saturation capacity and the exchange capacity efficiently by using the specific combinatorial structures that express the underlying polymatroids. But for the independent-flow problem as a general combinatorial optimization model, how to compute the saturation capacity and the exchange capacity efficiently had been left open, which is actually equivalent to minimizing submodular functions (in general) with a function evaluation oracle. This had long kept motivating me to try to devise an efficient algorithm for submodular function minimization.

The basic tools and algorithmic framework for the independent-flow problem are given in the paper [28], but the proposed algorithms are pseudopolynomial and terminates in finitely many steps when inputs are integral. The maximum independent-flow 
problem was first shown to be polynomially solvable by Schösleben [104] for polymatroid intersection and Lawler and Martel [85] for "polymatroidal flows." It is now known that these models are equivalent. For the currently fastest algorithms for such models see Fujishige and Zhang [68].

For the minimum-cost independent-flow problem I gave a "primal-dual"algorithm but, regrettably, I did not describe it in a form that used dual variables (potentials) explicitly as in [76] and [74] though I noticed that potentials could be used to make the algorithm faster as was mentioned for the independent-linkage problem [27]. Hence the results in [28] did not show the "total dual integrality" property of the independent-flow problem.

\subsection{Submodular flows}

When I finished the paper [28], I did not know the paper of Jack Edmonds and Rick Giles [18]. It came across to me through Uwe Zimmermann's working paper, published as [117]. Zimmermann employed techniques similar to the basic tools given in [28] and proposed an algorithm for submodular flows (also see [24] and [16]). It motivated me to examine the structure of the Edmonds-Giles (submodular flow) polyhedra.

After I finished [28], I got an idea on submodular function theory, which extends polymatroids ([30] and also see Section 4). The most important observation is that every polymatroid rank function $\rho: 2^{V} \rightarrow \mathbf{Z}_{+}$is monotone, submodular, and normalized $(\rho(\emptyset)=0)$ but the monotonicity does not play any essential role combinatorially. It only guarantees that the base polyhedron lies within the nonnegative orthant $\mathbf{R}_{+}^{V}$. So we should consider general but normalized, submodular functions (see Section 4).

Edmonds and Giles [18] originally formulated the submodular flow problem as follows. We are given a directed graph $G=(V, A)$ with vertex set $V$ and arc set $A$, lower and upper capacity functions $\underline{c}: A \rightarrow \mathbf{R} \cup\{-\infty\}$ and $\bar{c}: A \rightarrow \mathbf{R} \cup\{+\infty\}$ with $\underline{c} \leq \bar{c}$, a cost function $\gamma: A \rightarrow \mathbf{R}$, and a crossing-submodular function $f: \mathcal{F} \rightarrow \mathbf{R}$ on a crossing family $\mathcal{F} \subseteq 2^{V}$. (Here, for any $X, Y \in \mathcal{F}$, if all the four sets $X \backslash Y$, $Y \backslash X, X \cap Y$, and $V \backslash(X \cup Y)$ are nonempty, we say that $X$ and $Y$ cross, and $\mathcal{F} \subseteq 2^{V}$ is called a crossing family if for every crossing pair of $X, Y \in \mathcal{F}$ we have $X \cup Y \in \mathcal{F}$ and $X \cap Y \in \mathcal{F}$. A function $f: \mathcal{F} \rightarrow \mathbf{R}$ on a crossing family $\mathcal{F}$ is called crossing-submodular if the submodularity inequality

$$
f(X)+f(Y) \geq f(X \cup Y)+f(X \cap Y)
$$

holds for every crossing pair of $X, Y \in \mathcal{F}$.) Denote by $\mathcal{N}$ the network $(G, \underline{c}, \bar{c}, \gamma, f)$. A function $\varphi: A \rightarrow \mathbf{R}$ is called a submodular flow in $\mathcal{N}$ if it satisfies

$$
\sum_{a \in \Delta^{+} X} \varphi(a)-\sum_{a \in \Delta^{-} X} \varphi(a) \leq f(X) \quad(\forall X \in \mathcal{F}),
$$

where $\Delta^{+} X$ is the set of arcs leaving $X$ and $\Delta^{-} X$ that of $\operatorname{arcs}$ entering $X$. The submodular flow problem is to find a submodular flow $\varphi$ of minimum cost, where the 
cost is defined by

$$
\sum_{a \in A} \gamma(a) \varphi(a)
$$

(See [18]. The term, submodular flow, is due to Zimmermann [117].)

As can easily be seen, I noticed that the left-hand side of (10) is expressed as follows. Defining the boundary $\partial \varphi: V \rightarrow \mathbf{R}$ of flow $\varphi: A \rightarrow \mathbf{R}$ by

$$
\partial \varphi(v)=\sum_{a \in \delta^{+} v} \varphi(a)-\sum_{a \in \delta^{-} v} \varphi(a) \quad(v \in V)
$$

(10) is rewritten as

$$
\partial \varphi(X) \leq f(X) \quad(\forall X \in \mathcal{F})
$$

or equivalently

$$
\partial \varphi \in \mathrm{P}(f) \equiv\left\{x \in \mathbf{R}^{V} \mid \forall X \in \mathcal{F}: x(X) \leq f(X)\right\} .
$$

This reveals an important fact that since the boundary $\partial \varphi$ always satisfies $\partial \varphi(V)=0$, the relevant structure of $\mathrm{P}(f)$ in $(14)$ is the intersection of $\mathrm{P}(f)$ with the hyperplane $x(V)=0$. This motivated me to examine the structure of the polyhedron

$$
\mathrm{P}^{\prime}(f)=\{x \mid x \in \mathrm{P}(f), x(V)=0\} .
$$

Then I found that if $\mathrm{P}^{\prime}(f) \neq \emptyset$, it is a base polyhedron of a submodular system $(\mathcal{D}, \hat{f})$ [34] (see Section 4 for the definition of a submodular system). That is, if $\mathrm{P}^{\prime}(f) \neq \emptyset$, there uniquely exists a submodular system $(\mathcal{D}, \hat{f})$ on $V$ such that $\mathrm{P}^{\prime}(f)=\mathrm{B}(\hat{f})$. (I also showed a necessary and sufficient condition for the nonemptiness of $\mathrm{P}^{\prime}(f)$ in [34], but failed to give it as a theorem. I underestimated the importance of its implications but gratefully the credit was given to me by András Frank and others.)

Moreover, we can see that the set

$$
\partial \Phi \equiv\left\{\partial \varphi \mid \varphi \in \mathbf{R}^{A}, \forall a \in A: \underline{c}(a) \leq \varphi(a) \leq \bar{c}(a)\right\}
$$

of the boundaries of flows $\varphi$ between the lower and upper capacities is the base polyhedron associated with the cut function of the given network $\mathcal{N}$, where the cut function $\kappa_{\underline{c}, \bar{c}}: 2^{V} \rightarrow \mathbf{R} \cup\{+\infty\}$ is a submodular function defined by

$$
\kappa_{\underline{c}, \bar{c}}(X)=\sum_{a \in \Delta^{+} X} \bar{c}(a)-\sum_{a \in \Delta^{-} X} \underline{c}(a) \quad(X \subseteq V) .
$$

Hence if $\mathrm{P}^{\prime}(f) \neq \emptyset$, determining the feasibility of the submodular flow problem is equivalent to discerning whether the intersection of the two base polyhedra $\mathrm{B}(\hat{f})$ and $\partial \Phi$ is nonempty.

These observations brought us clear understanding of the essence of submodular flows. We can describe the submodular flow problem by means of an ordinary submodular function instead of a crossing-submodular function without loss of generality except for the total dual integrality of the relevant system of inequalities. Moreover, this revealed the 'equivalence' between the submodular flow model, the polymatroidal flow model, and the independent-flow model ([40]). 


\section{Theory of submodular functions}

After finishing [28] I was interested in finding practical problems having submodular structures. Tomizawa told me that the cores of convex games of Lloyd Shapley [106] are equivalent to polymatroids. A paper of Te-Sun Han [71] motivated me to think about a set of random variables and I found a "polymatroidal" structure on it. (While I was with Iri's laboratory, Han came to the laboratory very often to talk to Iri, Shun-ichi Amari, and others, and let me know of his result [71], a major part of his Ph.D. thesis.) I defined what is called the entropy function on the set of random variables, which is a typical example of a polymatroid and had not been recognized before as a submodular set function. I also looked for results in the journal of IEEE Transactions on Information Theory, with which I was familiar because of my Ph.D. study on filtering and estimation, and was excited to find Slepian and Wolf's 1973 paper [108] there. They treated two random variables separately sent to a common receiver and gave the region of "admissible" (noiseless coding) rates in a figure, which shows the submodular structure of the polymatroid on the two-element set (i.e., a two-dimensional supermodular polyhedron). Thomas Cover [13] also extended their result to the case of more than two random variables.

These findings resulted in a paper [29] on entropy functions, which extended a result of [71] and related ones and also treated the "principal partition" of polymatroids (see Section 5 below). More importantly, the paper [29] motivated me to extend polymatroids to submodular systems.

The set of admissible rates in [108] and [13] forms what is now called a supermodular polyhedron (or contra-polymatroid), which is an unbounded polyhedron consisting of spanning vectors of a polymatroid ( $x$ is spanning if $x \geq b$ for some base $b$ ). I used in [29] a then available concept of dual polymatroid with respect to a dominating vector shown in [91] ( $x$ is dominating if $x \geq b$ for every base $b$ ). A dual polymatroid is defined for each dominating vector, so that we do not have a concept of the dual polymatroid. Also note that if we consider unbounded base polyhedra, there is no dominating vector.

For a polymatroid $(V, \rho)$ with rank function $\rho: 2^{V} \rightarrow \mathbf{R}$ the polymatroid polytope $\mathrm{P}_{(+)}(\rho)$ is defined by (2). For any dominating vector $x$ define $\rho_{(x)}^{*}: 2^{V} \rightarrow \mathbf{R}$ by

$$
\rho_{(x)}^{*}(X)=x(X)+\rho(V \backslash X)-\rho(V) \quad(X \subseteq V) .
$$

We then have a dual polymatroid $\left(V, \rho_{(x)}^{*}\right)$.

On the other hand, the set of admissible rates corresponds to the unbounded polyhedron defined by the following inequalities ([13]).

$$
x(X) \geq \rho(V)-\rho(V \backslash X) \quad(X \subseteq V) .
$$

It would not be nice to say that this unbounded polyhedron and the polymatroid polytope are dual to each other.

Then I reached the following duality framework. Define the downward unbounded polyhedron

$$
\mathrm{P}(\rho)=\left\{x \in \mathbf{R}^{V} \mid \forall X \subseteq V: x(X) \leq \rho(X)\right\},
$$


which is called the submodular polyhedron associated with $(V, \rho)$. Note that the polymatroid polytope $\mathrm{P}_{(+)}(\rho)$ is the intersection of $\mathrm{P}(\rho)$ and the nonnegative orthant $\mathbf{R}_{+}^{V}$, and the two polyhedra $\mathrm{P}_{(+)}(\rho)$ and $\mathrm{P}(\rho)$ uniquely determine each other. Also define a set function by

$$
\rho^{\#}(X)=\rho(V)-\rho(V \backslash X) \quad(X \subseteq V),
$$

where $\rho^{\#}: 2^{V} \rightarrow \mathbf{R}$ is called the dual supermodular function of $\rho$ (the negative of a submodular function is called a supermodular function). Then the upward unbounded polyhedron given by (19) is expressed as

$$
\mathrm{P}\left(\rho^{\#}\right)=\left\{x \in \mathbf{R}^{V} \mid \forall X \subseteq V: x(X) \geq \rho^{\#}(X)\right\} .
$$

Moreover, define the base polyhedron by

$$
\mathrm{B}(\rho)=\{x \mid x \in \mathrm{P}(\rho), x(V)=\rho(V)\} .
$$

It should be noted that $\mathrm{B}(\rho)$ here coincides with the base polytope of the polymatroid defined by (3) when $\rho$ is a polymatroid rank function.

We can regard a supermodular function as a submodular function with respect to the dual order $\leq^{*}$ of the order $\leq$ of the underlying totally ordered additive group $\mathbf{R}$ $\left(a \leq b \Leftrightarrow b \leq^{*} a\right.$ ). In this sense it is natural to consider a one-to-one correspondence between the set of submodular functions $\rho$ and that of dual supermodular functions $\rho^{\#}$. With respect to the dual order $\leq^{*}$ the base polyhedron associated with $\rho^{\#}$ is given by

$$
\mathrm{B}\left(\rho^{\#}\right)=\left\{x \mid x \in \mathrm{P}\left(\rho^{\#}\right), x(V)=\rho^{\#}(V)\right\} .
$$

We can easily see that $\mathrm{B}\left(\rho^{\#}\right)=\mathrm{B}(\rho)$. (Originally I used symbol $*$ instead of \# in [30]; I borrowed it from Tomizawa in 1980 since symbol $*$ is often used for other operations in mathematics. )

We now have a nice duality and, more importantly, this duality framework works for all normalized submodular functions and supermodular functions. We do not require the monotonicity of $\rho$, which is imposed on polymatroid rank functions. It should be noted that a translation of $\mathrm{P}(\rho)$ or $\mathrm{B}(\rho)$ does not change the combinatorial structure of the polyhedron but the translation results in an addition of the associated modular function to $\rho$, which may destroy the monotonicity of the submodular function that determines the translated polyhedron.

Through the investigation of independent flows and principal partitions I found it convenient to consider the domains of submodular functions not as Boolean lattice $2^{\mathrm{V}}$ but as distributive lattices $\mathcal{D} \subseteq 2^{V}$ closed with respect to set union and intersection. Given a distributive lattice $\mathcal{D} \subseteq 2^{V}$ with $\emptyset, V \in \mathcal{D}$ and a submodular function $f$ : $\mathcal{D} \rightarrow \mathbf{R}$ with $f(\emptyset)=0$, we call the pair $(\mathcal{D}, f)$ a submodular system on $V$. The submodular polyhedron associated with the submodular system $(\mathcal{D}, f)$ is defined by

$$
\mathrm{P}(f)=\left\{x \in \mathbf{R}^{V} \mid \forall X \in \mathcal{D}: x(X) \leq f(X)\right\},
$$

and the base polyhedron by

$$
\mathrm{B}(f)=\{x \mid x \in \mathrm{P}(f), x(V)=f(V)\} .
$$


We define a supermodular system, the associated supermodular polyhedron, and the base polyhedron in a similar and dual manner. The dual supermodular system $\left(\overline{\mathcal{D}}, f^{\#}\right)$ of submodular system $(\mathcal{D}, f)$ is given by

$$
\begin{aligned}
& \overline{\mathcal{D}}=\{V \backslash X \mid X \in \mathcal{D}\}, \\
& f^{\#}(V \backslash X)=f(V)-f(X) \quad(X \in \mathcal{D}) .
\end{aligned}
$$

Note that under the correspondence between $X \in \mathcal{D}$ and $V \backslash X \in \overline{\mathcal{D}}$ the two distributive lattices are dual lattices. Similarly as discussed above, we have

$$
\mathrm{B}(f)=\mathrm{B}\left(f^{\#}\right), \quad\left(f^{\#}\right)^{\#}=f .
$$

It is often said in the literature that it suffices to consider submodular functions $f$ on Boolean lattices by putting $f(X)=+\infty$ for those $X$ not in the domain (distributive lattice) of $f$. But explicitly considering the underlying distributive lattice makes it easier for us to investigate the combinatorial structure of the associated polyhedra. For example, the base polyhedron $\mathrm{B}(f)$ is pointed if and only if the length of a maximal chain of the distributive lattice $\mathcal{D}$ is equal to $|V|$. It is also interesting to see from the greedy algorithm ([17] and [64]) that when $\mathrm{B}(f)$ is pointed, every extreme point $b \in \mathrm{B}(f)$ satisfies the following. For any $u \in V$, letting $X_{u}$ be the unique maximal $X \in \mathcal{D}$ with $u \notin X$ and $X^{u}$ the unique minimal $X \in \mathcal{D}$ with $u \in X$, we have

$$
f\left(X_{u} \cup\{u\}\right)-f\left(X_{u}\right) \leq b(u) \leq f\left(X^{u}\right)-f\left(X^{u} \backslash\{u\}\right) .
$$

Define $\underline{\alpha}, \bar{\alpha} \in \mathbf{R}^{V}$ by

$$
\underline{\alpha}(u)=f\left(X_{u} \cup\{u\}\right)-f\left(X_{u}\right), \quad \bar{\alpha}(u)=f\left(X^{u}\right)-f\left(X^{u} \backslash\{u\}\right) \quad(u \in V) .
$$

We see that $\underline{\alpha}$ is nonnegative (i.e., all the extreme bases lie in the nonnegative orthant) if and only if $f$ is monotone.

This reminds me of the Oberwolfach meeting held in January, 1983 (my talk given there appeared in [35]). In an evening of the meeting after dinner some participants gathered in a room and began to exchange comments on submodular functions. László Lovász, András Frank, Éva Tardos, Tom Liebling and others were there and discussing submodular function minimization. One of the questions we discussed there was how to discern the monotonicity of a set function. Under submodularity the question can be answered by the nonnegativity of $\underline{\alpha}$ defined above. I also met Peter Hammer at the meeting, who later gave me a chance to write the monograph [45].

I have further developed a theory of submodular functions based on the duality framework. For more details see [45] (also see Sections 5 and 6 below).

There are several important subjects related to submodularity but not included in full details in [45]. The subject on bisubmodular functions is one of them. I worked on bisubmodular functions with my then doctoral students Takeshi Naitoh, Toshio Nemoto, and Kazutoshi Ando ([2], [3], [5], [6], [7]) and with Sachin Patkar [59]. I also found a nice min-max theorem for bisubmodular functions [41], which gave a basis for bisubmodular function minimization (see joint work with Iwata [54] and with Tom McCormick [90]). Also an extension of [3] to jump systems appeared in [4]. 


\section{Principal Partitions}

The theory of principal partitions was inaugurated in Japan in 1967 by Genya Kishi and Yoji Kajitani [83] (see [75] and [46] for details about its history and developments until recently).

When I began to work with Iri in 1975, the theory of principal partitions reached the peak for matroids, which was established independently by Tomizawa [111] and H. Narayanan [97]. The essence of their results is the following. For any matroid $\mathbf{M}=(V, \rho)$ with rank function $\rho$ there uniquely exists a sequence of rationals

$$
\frac{k_{1}}{\ell_{1}}<\frac{k_{2}}{\ell_{2}}<\cdots<\frac{k_{p}}{\ell_{p}}
$$

with positive integers $k_{i}$ and $\ell_{i}(i=1, \cdots, p)$ and a partition

$$
\left\{U_{1}, U_{2}, \cdots, U_{p}\right\}
$$

of $V$ such that every element of the underlying set $U_{i}$ of each minor $\mathbf{M} \cdot S_{i} / S_{i-1}$ for $i=1,2, \cdots, p$ with $S_{i}=\cup_{j=1}^{i} U_{j}$ and $S_{0}=\emptyset$ is uniformly covered $k_{i}$ times by $\ell_{i}$ bases of the minor. (Here $\mathbf{M} \cdot S_{i} / S_{i-1}$ is the restriction of $\mathbf{M}$ to $S_{i}$ followed by the contraction by $S_{i-1}$, which is a matroid on $U_{i}=S_{i} \backslash S_{i-1}$.) The decomposition of matroid $\mathbf{M}$ into such minors $\mathbf{M} \cdot S_{i} / S_{i-1}(i=1,2, \cdots, p)$ is called the principal partition of matroid $\mathbf{M}$.

Through the research on independent flows I noticed that the principal partition of matroids can better be understood in terms of polymatroid. That is, if we consider a given matroid $\mathbf{M}=(V, \rho)$ as a polymatroid $\mathbf{P}=(V, \rho)$, then the above condition for the principal partition is equivalent to that there uniquely exists a base $b^{*}$ of polymatroid $\mathbf{P}$ such that the distinct values of $b^{*}(u)(u \in V)$ are given by (32) and, defining $U_{i}=\left\{u \in V \mid b^{*}(u)=\frac{k_{i}}{\ell_{i}}\right\}$ and $S_{i}$ as above, for any $i \in\{1,2, \cdots, p-1\}$ and any $\lambda \in \mathbf{R}$ with $\frac{k_{i}}{\ell_{i}} \leq \lambda<\frac{k_{i+1}}{\ell_{i+1}}$ we have

$$
\operatorname{sat}\left(b^{*} \wedge \lambda \mathbf{1}\right)=S_{i},
$$

where $\left(b^{*} \wedge \lambda \mathbf{1}\right)(u)=\min \left\{b^{*}(u), \lambda\right\}$ for all $u \in V$. For such a base $b^{*}$ construct the exchangeability graph $G_{b^{*}}=\left(V, A_{b^{*}}\right)$ by

$$
A_{b^{*}}=\left\{(u, v) \mid u, v \in V, v \in \operatorname{dep}\left(b^{*}, u\right) \backslash\{u\}\right\} .
$$

Then the decomposition of $G_{b^{*}}$ into strongly connected components gives the principal partition of $\mathbf{M}$. This gives an even more finer decomposition (than the one described above) together with the poset structure on the partition, which was already examined by Tomizawa and Narayanan. I also found that Megiddo's lexicographically optimal flow [92] corresponds to the base $b^{*}$ in the principal partition of a polymatroid induced on the set of entrances (or exits) of the network. This observation lead us to a faster algorithm for the lexicographically optimal flow problem [31], which was further improved by Gallo, Grigoriadis, and Tarjan [69]. 
The way of understanding the principal partition as described above opened up a new vista from the point of view of submodular function theory by extending the roles of base $b^{*}$ and parametric vector $\lambda \mathbf{1}$. Moreover, through discussions with Fumihiro Maruyama during his graduation study in 1977-78 we noticed that $b^{*}$ is the minimumnorm base (with respect to the Euclidean norm) of the associated base polyhedron. This revealed the relationship between the principal partition and a resource allocation problem with submodular constraints (see [31], [46]). It also revealed that we can apply Philip Wolfe's minimum-norm point algorithm [116] to find the minimumnorm base $b^{*}$ because Wolfe's algorithm works for polytopes over which linear optimization is easy. For a general submodular system $(\mathcal{D}, f)$ equation (34) also holds and if $\frac{k_{i}}{\ell_{i}} \leq 0<\frac{k_{i+1}}{\ell_{i+1}}, S_{i}$ is a (unique maximal) minimizer of $f$ (also see Section 7.1 below).

For more details and recent work with Kiyohito Nagano and others see [46], [58], and [48].

\section{Submodular Analysis}

In early 1980's Lovász [86] and Frank [24] pointed out the relationship and analogy between submodular functions and ordinary convex functions. Lovász gave a plenary talk at the ISMP (International Symposium on Mathematical Programming) in Bonn in August, 1982, where one of the theorems he talked about was the following: a set function is submodular if and only if its (certain) extension (now called the Lovász extension) is convex. (But there is also a good reason to regard submodularity as concavity.)

Tomizawa and I were hearing his ISMP talk in Bonn, which reminded us of an argument between us made before the ISMP. We discussed whether submodular functions are convex or concave. Tomizawa was on the concavity side and I was on the convexity side. My intuition was based upon the 'fact' that whenever a submodular function appears in a min-max theorem, it always appears in the minimum side, so that minimization is meaningful for submodular functions, which is the case for convex functions. I had also obtained a min-max relation for submodular function minimization through a min-max relation for independent flows and that for principal partitions. So I felt the analogy between submodular functions and convex functions, and examined it (see [35], [36], [37], and [38]). The following observation initiated my research on this topic.

The system of inequalities for the submodular polyhedron $\mathrm{P}(f)$ associated with a submodular system $(\mathcal{D}, f)$ on $V$ is given by

$$
x(X) \leq f(X) \quad(X \in \mathcal{D}) .
$$

It is interesting to see that identifying each $X \in \mathcal{D}$ with its characteristic vector $\chi_{X} \in \mathbf{R}^{V}$, we can rewrite (36) as

$$
\left\langle x, \chi_{X}-\chi_{\emptyset}\right\rangle \leq f\left(\chi_{X}\right)-f\left(\chi_{\emptyset}\right) \quad(X \in \mathcal{D}),
$$


where $\langle x, y\rangle=\sum_{u \in V} x(u) y(u)$. By analogy to the concept of a subgradient of an ordinary convex function ([101]) inequality (37) suggests that we should call a vector $x \in \mathrm{P}(f)$ a subgradient of $f$ at $\emptyset$. In this way of thinking let us define a subgradient of $f$ at $Y \in \mathcal{D}$ as a vector $x \in \mathbf{R}^{V}$ that satisfies

$$
x(X)-x(Y) \leq f(X)-f(Y) \quad(X \in \mathcal{D}) .
$$

Denote by $\partial f(Y)$ the set of all the subgradients (subdifferential) of $f$ at $Y \in \mathcal{D}$. Hence we have $\mathrm{P}(f)=\partial f(\emptyset)$. We can also show that $\mathbf{0} \in \partial f(Y)$ if and only if $Y$ is a minimizer of $f$, similarly as in the ordinary convex analysis (see [37]).

This further motivated me to define the (convex) conjugate function $f^{*}: \mathbf{R}^{V} \rightarrow$ $\mathbf{R}$ by

$$
f^{*}(y)=\max \{y(X)-f(X) \mid X \in \mathcal{D}\} \quad\left(y \in \mathbf{R}^{V}\right) .
$$

This is an ordinary convex function on $\mathbf{R}^{V}$. Then for a submodular system $(\mathcal{D}, f)$ and a supermodular system $(\mathcal{D}, g)$ on $V$ we have a Fenchel-type min-max duality as

$$
\min \{f(X)-g(X) \mid X \in \mathcal{D}\}=\max \left\{g^{*}(y)-f^{*}(y) \mid y \in \mathbf{R}^{V}\right\},
$$

where if $f$ and $g$ are integer-valued, the maximum in the right-hand side is attained by an integral vector $y$ (see [36]). (Here, the concave conjugate function $g^{*}$ of a supermodular function $g$ is defined by (39) with $f$ replaced by $g$ and max by min.) It turned out ([36]) that the min-max relation (40) is equivalent to Edmonds' intersection theorem [17] and to Frank's discrete separation theorem [24].

Moreover, we can consider the convex conjugate of $f^{*}$ as follows.

$$
\left(f^{*}\right)^{*}(x)=\sup \left\{\langle x, y\rangle-f^{*}(y) \mid y \in \mathbf{R}^{V}\right\} \quad\left(x \in \mathbf{R}^{V}\right) .
$$

Then $\left(f^{*}\right)^{*}$ is (essentially) what is called the Lovász extension of $f$ (see [86]). The subdifferential $\partial f(X)$ of $f$ at $X \in \mathcal{D}$ is equal to the ordinary subdifferential of $\left(f^{*}\right)^{*}$ at $\chi_{X}$.

A submodular function $f: \mathcal{D} \rightarrow \mathbf{R}$ can thus be regarded as a 'discrete convex' function on a 'discrete convex' domain $\mathcal{D}$ (also see [38]). I called the research paradigm along this direction submodular analysis, taking after convex analysis of Rockafellar [101] (see [45, Chapter IV]).

Kazuo Murota [94] established the theory of discrete convex analysis that includes submodular analysis as a very special case. Function $f^{*}$ in (39) is an example of $\mathrm{M}^{\natural}$-convex function.

\section{Submodular Function Minimization}

The problem of submodular function minimization (SFM) has been one of my major research subjects since 1977 after completing the paper [28]. As we mentioned in Section 5, the research on the principal partition of (poly)matroids brought us an algorithm for SFM by means of the minimum-norm base, to which we can apply the minimum-norm point algorithm of Wolfe [116]. 
7.1 Minimum-norm base and SFM

Suppose that we are given the minimum-norm base $b^{*}$ of a submodular system $(\mathcal{D}, f)$ on $V$. Let us compute

$$
\begin{aligned}
& A_{-}=\left\{u \in V \mid b^{*}(u)<0\right\} \\
& A_{0}=\left\{u \in V \mid b^{*}(u) \leq 0\right\} .
\end{aligned}
$$

Then $A_{-}$is the unique minimal minimizer and $A_{0}$ is the unique maximal minimizer of $f$.

The original Wolfe algorithm is designed to find the minimum-norm point in a polytope expressed as the convex hull of input vectors. A base polytope (a bounded base polyhedron) can have $|V|$ ! extreme points, so that apparently any direct application of the Wolfe algorithm seems to be difficult. But the important thing is that the Wolfe algorithm works if the linear optimization over the polytope can easily be carried out: this is the case for base polytopes by means of the greedy algorithm. I mentioned this at the 1983 Oberwolfach meeting.

I offered the subject of applying the Wolfe algorithm to SFM to some of my undergraduate students as research subjects of their graduation theses. Masaharu Nakayama carried out computational experiments for minimum covers of bipartite graphs in 1984-85, Shingo Shikita did it for minimum cuts of capacitated networks in 1986-87, and so on, which showed that the minimum-norm base algorithm for SFM runs fairly fast and could be a good algorithm for SFM. However, we considered it as a practically efficient but not polynomial algorithm such as the simplex method for linear programming.

It was after polynomial combinatorial algorithms were devised ([80], [105]) that I really tried hard to make the minimum-norm base algorithm for SFM a practically efficient code with the help of Shigueo Isotani (see [49] and [52], and a code in C for SFM is now available upon request).

It is an open question to decide whether the minimum-norm base algorithm for SFM is a polynomial one or not. Moreover, there are many possible ways of modifying the minimum-norm base algorithm for SFM from the point of view of computational efficiency, which is still being one of my research subjects.

\subsection{Combinatorial polynomial SFM algorithm}

I moved from University of Tokyo to University of Tsukuba in August, 1979, and further moved to Osaka University in April, 1997. I was so happy that I was allowed to invite two young brilliant scholars, Satoru Iwata and Kazuhisa Makino, to my laboratory and they could join me from the beginning of April, 1997 there.

I found Iwata's 1997 paper [78] very interesting and felt that his scaling algorithm could be adapted to yield a polynomial SFM algorithm. So I proposed to him a joint project on SFM algorithms. The first joint work is [53]. But intensive joint work really started in January, 1999 and it took about four months to obtain a combinatorial 
weakly polynomial SFM algorithm and about two more months to make it strongly polynomial. Before we completed our paper, we had proposed Lisa Fleischer to join our project since we had employed her idea arisen in a discussion between Lisa and Iwata concerning submodular flow algorithms.

I was very happy to hear from Bill Cunningham in July, 1999 that he received almost simultaneously two manuscripts, one being ours already submitted to JACM and the other being Lex Schrijver's, which independently solved the same long-standing open problem. Both algorithms are based on Cunningham's framework ([14], [15]) of expressing a base as a convex combination of extreme bases but otherwise they are quite different ([80], [105]).

The original version of our algorithm relied on a procedure for constructing partially ordered sets associated with extreme bases developed by Bixby, Cunningham, and Topkis [11]. We later improved our algorithm by getting rid of this procedure while Lisa and Iwata were staying at the Fields Institute in Toronto.

Iwata has achieved further algorithmic developments in SFM. See [79] for details about the developments due to him and others. Currently the fastest SFM algorithm is James Orlin's [99] (also see a recent algorithm [81]).

I am still not satisfied with the present status of SFM algorithms. I would like to find a more 'genuinely combinatorial' SFM algorithm.

\subsection{Combinatorial hull}

Schrijver's algorithm for SFM is based on the following key fact. Given an extreme base $y$ that corresponds to a linear ordering $L=\left(v_{1}, v_{2}, \cdots, v_{n}\right)$ of the underlying set $V$, denote by $\leq_{L}$ the linear order corresponding to $L$ on $V$, consider two elements $s, t \in V$ with $v_{1} \leq_{L} s<_{L} t \leq_{L} v_{n}$, and define an interval $(s, t]_{L}$ by

$$
(s, t]_{L}=\left\{v \in V \mid s<_{L} v \leq_{L} t\right\} .
$$

For each $u \in(s, t]_{L}$ let $L^{s, u}$ be the linear ordering of $V$ obtained from $L$ by moving $u$ to the position immediately before $s$ and denote by $\leq_{L}^{s, u}$ the linear order corresponding to $L^{s, u}$. Also denote by $y^{s, u}$ the extreme base determined by the greedy algorithm, associated with the linear ordering $L^{s, u}$. Then, from the submodularity of $f$ we can easily see the following. For each $u \in(s, t]_{L}$ we have

$$
y_{L}^{s, u}(v)-y(v)= \begin{cases}- & \text { if } s \leq_{L} v<_{L} u \\ + & \text { if } v=u \\ 0 & \text { otherwise, }\end{cases}
$$

where $z=-$ means $z \leq 0$ and $z=+$ means $z \geq 0$. Then for a (unique) $\alpha>0$, $\alpha\left(\chi_{t}-\chi_{s}\right)$ is expressed as a convex combination of $y_{L}^{s, u}-y_{L}\left(u \in(s, t]_{L}\right)$, i.e.,

$$
\alpha\left(\chi_{t}-\chi_{s}\right)=\sum_{u \in(s, t]_{L}} \mu_{u}\left(y_{L}^{s, u}-y_{L}\right)
$$


with $\mu_{u}>0\left(u \in(s, t]_{L}\right)$ and $\sum_{u \in(s, t]_{L}} \mu_{u}=1$. Note that (46) is rewritten as

$$
y_{L}+\alpha\left(\chi_{t}-\chi_{s}\right)=\sum_{u \in(s, t]_{L}} \mu_{u} y_{L}^{s, u}
$$

This means that $y_{L}$ can be transformed into a new base by exchanging from $s$ to $t$ by the amount of $\alpha$, i.e., $0<\alpha \leq \tilde{\mathrm{c}}\left(y_{L}, t, s\right)$. Schrijver's algorithm repeatedly applies possible such elementary transformations (47) to extreme bases that express a current base as their convex combination, to reduce the interval $(s, t]_{L}$. This makes the number of extreme bases expressing a current base grow exponentially, so that we need to repeatedly reduce the number of extreme bases by the Gaussian elimination.

Now, compare the two (extreme) bases $y_{L}$ and $y_{L}^{s, t}$. We have

$$
\begin{array}{ll}
y_{L}(u) \leq y_{L}^{s, t}(u), & (u=t), \\
y_{L}(u) \geq y_{L}^{s, t}(u) & \left(s \leq_{L} u<_{L} t\right), \\
y_{L}(u)=y_{L}^{s, t}(u) & \text { (otherwise). }
\end{array}
$$

We can easily see that the following "box" region $B\left(y_{L}, y_{L}^{s, t}, t\right)$ is contained in the base polyhedron $\mathrm{B}(f)$.

$$
\begin{aligned}
& B\left(y_{L}, y_{L}^{s, t}, t\right)=\left\{x \in \mathbf{R}^{V} \mid\right. x(V)=f(V), y_{L}(t) \leq x(t) \leq y_{L}^{s, t}(t) \\
&\left.\forall u \in V \backslash\{t\}: y_{L}(u) \geq x(u) \geq y_{L}^{s, t}(u)\right\}
\end{aligned}
$$

In particular, if $y_{L}^{s, t}(s)<y_{L}(s)$, then putting $\alpha^{\prime}=y_{L}(s)-y_{L}^{s, t}(s)$, we have

$$
y_{L}+\alpha^{\prime}\left(\chi_{t}-\chi_{s}\right) \in \mathrm{B}(f) .
$$

That is, we have a 'valid' elementary transformation (50) without using any linear algebraic computation (Gaussian elimination), which is required in Schrijver's algorithm.

It should be noted that the above argument in (48) (50) holds for any two bases $y$ and $y^{\prime}$ such that $y(t)>y^{\prime}(t)$ and $y(u) \leq y^{\prime}(u)$ for all $u \in V \backslash\{t\}$. Denote by $B\left(y, y^{\prime}, t\right)$ the "box" region defined by (49) with $y_{L}$ and $y_{L}^{s, t}$ being replaced by $y$ and $y^{\prime}$, respectively. We call $B\left(y, y^{\prime}, t\right)$ the combinatorial hull of the two bases $y$ and $y^{\prime}$, and each $x \in B\left(y, y^{\prime}, t\right)$ a combinatorial combination of $y$ and $y^{\prime}$. Note that we need only addition, subtraction, and comparison for the combinatorial hull and combinatorial combination. (I gave a talk on "Combinatorial hull and submodular function minimization" at the ISMP held in Atlanta in August, 2000.)

We do not know whether we can effectively incorporate the combinatorial hull into Schrijver's algorithm and whether we can construct a 'genuinely' combinatorial polynomial algorithm by means of combinatorial hull. We do not even know how many (possibly polynomially many) times we should take combinatorial combination to prove the membership in the base polyhedron of any given point by using some extreme bases, a kind of Carathéodory theorem.

To devise a polynomial SFM algorithm by means of combinatorial hull (or something genuinely combinatorial) has been one of the most important research subjects to me. 
8 LP-Newton Method for Linear Programming Problems

While applying Wolfe's minimum-norm point algorithm to SFM, I was getting interested both in Wolfe's algorithm and in the minimum-norm point problem itself. I enjoyed research on related problems and algorithms as joint work with my students ([56], [61], [66], [67]).

After resolving the problem of constructing a combinatorial polynomial algorithm for SFM, my research interests slightly shifted to the open problem of constructing a strongly polynomial algorithm for linear programming problems (or of disproving the existence of such an algorithm). Then I noticed applicability of the minimum-norm point algorithm to linear programming problems in the following way.

Let us consider a linear programming problem (LP) given by

$$
\begin{gathered}
\text { (LP) Maximize }\langle c, x\rangle=\sum_{j=1}^{n} c(j) x(j) \\
\text { subject to } A x=b, \\
l \leq x \leq u,
\end{gathered}
$$

where $A$ is an $m \times n$ real matrix, $b$ an $m$-dimensional column vector in $\mathbf{R}^{m}, l$ and $u n$-dimensional column vectors, $x$ an $n$-dimensional column variable vector in $\mathbf{R}^{n}$, and $c$ an $n$-dimensional row vector in $\left(\mathbf{R}^{n}\right)^{*}$. Here note that there is a bounded box constraint in our model, which is not a crucial assumption either theoretically or practically.

Now define an $(m+1) \times n$ matrix

$$
\bar{A}=\left(\begin{array}{c}
A \\
c
\end{array}\right)
$$

and a polytope

$$
\bar{Z}=\{\bar{z} \mid \bar{z}=\bar{A} x, l \leq x \leq u\} .
$$

Then problem (LP) can be reduced to

$$
\begin{array}{ll}
(\mathrm{LP})^{\prime} \quad \text { Maximize } & \gamma \\
& \text { subject to }\left(\begin{array}{l}
b \\
\gamma
\end{array}\right) \in \bar{Z},
\end{array}
$$

where $\gamma$ is a scalar variable in $\mathbf{R}$. Also define a line

$$
L=\left\{\left(\begin{array}{l}
b \\
\gamma
\end{array}\right) \mid \gamma \in \mathbf{R}\right\} .
$$

Note that $L$ is a line parallel to the last coordinate space of $\mathbf{R}^{m+1}$. 
It should be noted that the polytope $\bar{Z}$ is what is called a zonotope and that linear optimization over every zonotope is easily done in a greedy way. Hence $\bar{Z}$ is a polytope for which Wolfe's minimum-norm point algorithm works. The reduced (LP)' is to find the point in the intersection of the line $L$ and the zonotope $\bar{Z}$ whose last coordinate value is as large as possible (see Figure 1).

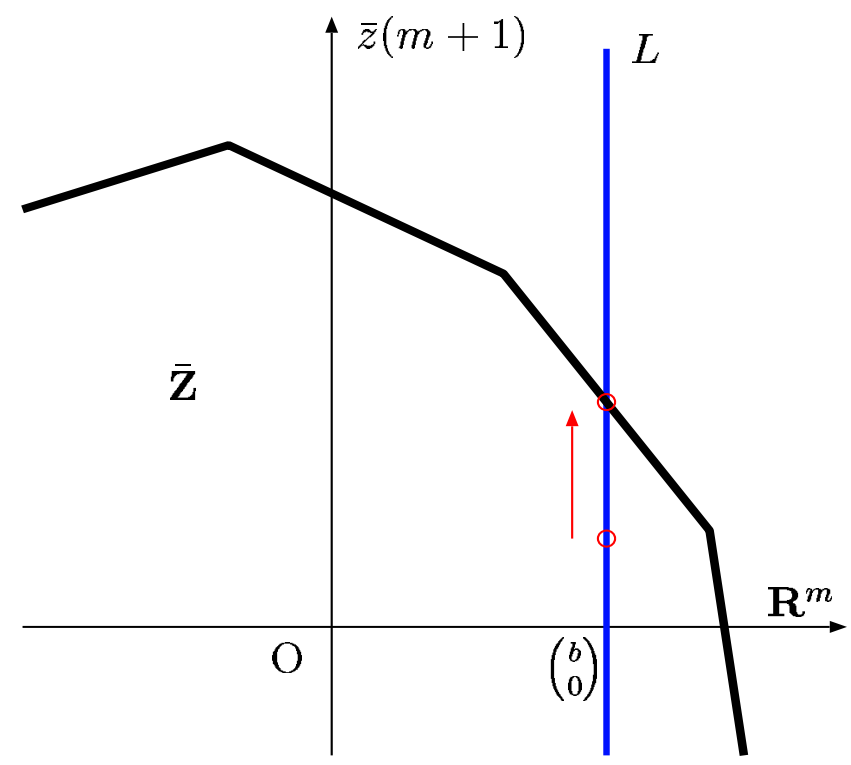

Figure 1: A primal view

Moreover, we can view problem (LP) ${ }^{\prime}$ in a dual manner as follows. Consider a hyperplane that supports the zonotope $\bar{Z}$ from above along the last coordinate and find the intersection point of $L$ and the hyperplane (see Figure 2). 


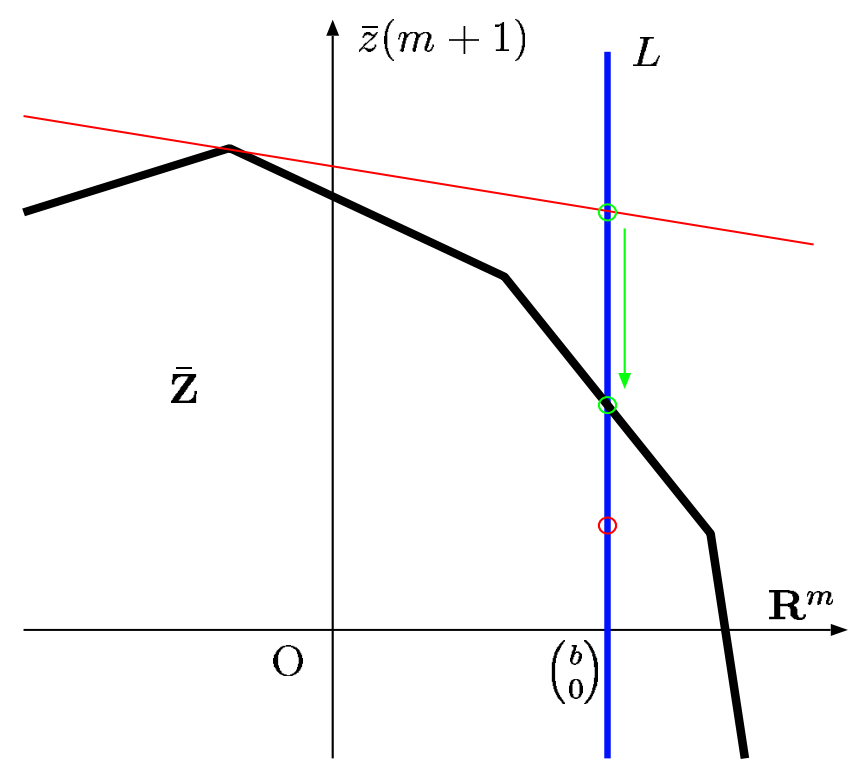

Figure 2: A dual view

This dual view suggests us to use Wolfe's algorithm as shown in Figure 3. The initial supporting hyperplane has the normal vector in the direction of the last coordinate, $\bar{b}_{0}$ is the intersection point of the hyperplane and $L, z_{1}$ is the point of $\bar{Z}$ nearest to $\bar{b}_{0}$, the next supporting hyperplane $H_{z_{1}}$ supports $\bar{Z}$ at $z_{1}$ with a normal vector $\bar{b}_{0}-z_{1}$, and so on. This process is similar to the Newton method, and is a higher dimensional variant of the Newton method that finds an end point of the intersection of the line $L$ and the polytope (zonotope) $\bar{Z}$. Hence we call this algorithm the LP-Newton method.

It should be noted that the LP-Newton method can be applied to any polytope over which linear optimization can easily be made, to find an end point of the intersection of a line and the polytope. See [50] for preliminary computational results. There are many possible ways of modifying the original LP-Newton method. The present status is far from being satisfactory. I hope that the LP-Newton method would stimulate research toward finding a strongly polynomial algorithm for LP and, if not strongly polynomial, a new efficient algorithm other than the interior-point method and the simplex method. 


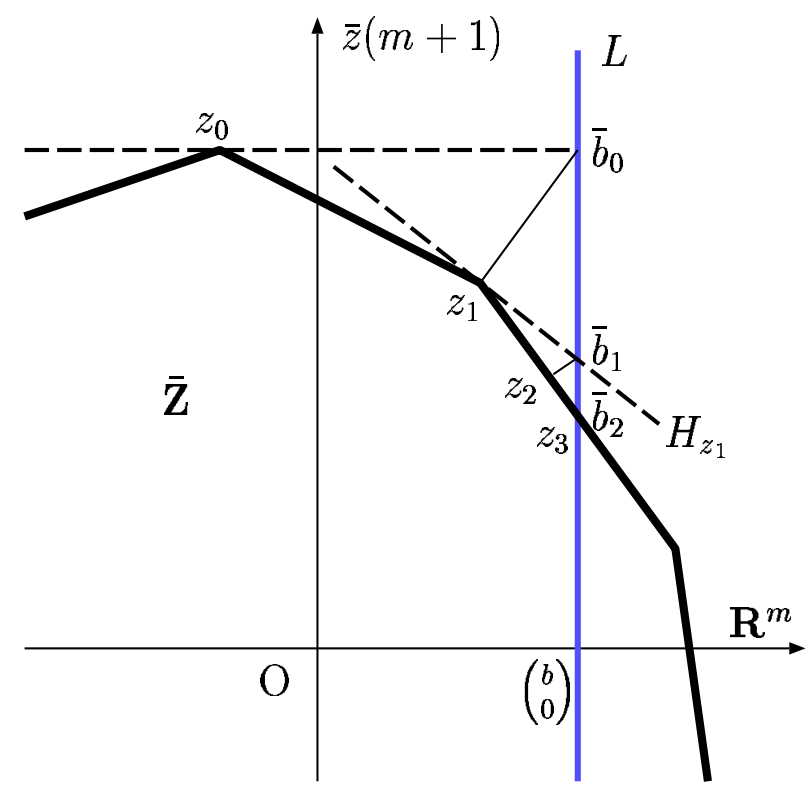

Figure 3: An illustration of the LP-Newton method [50]

\section{The Graph-Realization Problem}

The graph-realization problem is to discern whether a given $\{0,1\}$-matrix $N$ is graphic (i.e., $N$ is a fundamental circuit matrix of a graph) and if graphic, to realize a graph that has $N$ as its fundamental circuit matrix. The problem is fundamental both theoretically and practically. It is related to problems such as identification of graphic or cographic (and hence regular) matroids ([113], [114]), synthesis of electric networks ([73], [100]), and extraction of network flow structures in linear programming problems ([73], [10]).

Around 1960 there were a number of methods proposed for solving the graphrealization problem, among which was William Tutte's algorithm [114]. But then existing algorithms were hardly regarded as polynomial ones since the theory of algorithms and data structures had not been developed till then. The first polynomial algorithm for the graph-realization problem was given by Iri [73] based on linear algebraic procedures.

In 1976 Tomizawa devised an $\mathrm{O}\left(\mathrm{m}^{3}\right)$ algorithm [112] improving Iri's, where $m$ is the number of columns of a given matrix $N$. Early in 1979 I presented an algorithm at a seminar of Iri's laboratory, which required a subroutine to maintain a matrix of consecutive one's property. Iri introduced me Booth and Lueker's data structure, $P Q$ tree, given in 1976. Using $P Q$-trees, I proposed the data structure of $P Q$-graph and gave an almost-linear time algorithm for the graph-realization problem [32], almost- 
linear in the number of non-zero elements of $N$, which is of the best complexity though it lacks detailed description in some part and has some minor correctable bugs. Later Bob Bixby and Donald Wagner [12] also gave an algorithm of the same complexity based on the Tutte decomposition of graphs [115]. (The idea of using the Tutte decomposition was also pointed out by Tomizawa after my paper [32] but has not been published.) See experimental results by Takahiro Ohto in [98] and his 2002 master's thesis at University of Tokyo (also see [1]).

When I was completing the manuscript of [32], Iri showed me a preprint of Bixby and Cunningham [10]. They presented a polynomial graph-realization algorithm based on a matroid decomposition, and used it to convert linear programming problems to network flow problems, which is the same subject as Iri considered in 1968 [73]. Bob Bixby really concentrated on implementing their algorithm to linear programs in collaboration with Robert Fourer and others, which lead them to construct a software for linear programs. This is exactly the origin of Bob's pathway to CPLEX and recent GUROBI.

\section{Network Optimization}

While I have been interested in the submodular flow problem as mentioned above, I have also been interested in the classical network optimization problems such as shortest paths, maximum flows, min-cost flows, and generalized flows.

\subsection{Shortest paths}

I have only one paper dealing with the shortest path problem [33], which proposed a shortest-path algorithm for networks with arcs of negative length.

As is well-known, the shortest path problem with negative arcs usually requires more computation time than that without negative arcs. Consider a (directed) graph $G=(V, A)$ and a length function $\ell: A \rightarrow \mathbf{R}$ with possible arcs of negative length. Suppose that we have solved the shortest path problem for the network $\mathcal{N}=(G, \ell)$. We then have a potential $p: V \rightarrow \mathbf{R}$ on the vertex set $V$ of the network such that every arc length $\ell(a)$ can be transformed into a nonnegative one by putting

$$
\ell_{p}(a)=\ell(a)+p\left(\partial^{+} a\right)-p\left(\partial^{-} a\right) \quad(a \in A)
$$

where $\partial^{+} a$ (resp. $\partial^{-} a$ ) is the initial (resp. terminal) vertex of $\operatorname{arc} a$.

If we are given such a potential (called a feasible potential) beforehand, then the shortest path problem can be solved by faster Dijkstra's algorithm using the transformed nonnegative length function $\ell_{p}$. Any real-valued feasible potential $p$ works for this. Hence, there arises a question: Can we find a feasible potential faster than solving the original shortest path problem with possible negative arcs? This has long been a big question to which I want to get an answer. 


\subsection{Network flows}

\section{Min-cost flows}

It was a great surprise when Iri showed me a working paper in February (or January) 1985. It was Tardos's working paper on a strongly polynomial algorithm for min-cost flows (see [110]). I was very much impressed with her elegant approach to the mincost flows by cost rounding. She employed the orthogonal projection of a cost vector into the circulation space (which should be regarded as the projection to the orthogonal complement of the tension space), so that her strongly polynomial algorithm requires linear algebraic computation such as the Gaussian elimination.

I felt that we could do it more combinatorially, and found that a spanning tree could work to extract useful information about the invariant cycle lengths for fundamental cycles with respect to potential changing. This gave a (combinatorial) algorithm having essentially the same complexity as hers. So I tried to take a dual approach by means of a spanning tree and its fundamental cutsets for circulations, and obtained a faster algorithm [39]. Before the publication of [39] I heard from Bill Cunningham that (in some conference in North America) there were discussions on Tardos's algorithm and a question was posed about how to make her algorithm more combinatorial, i.e., how to get rid of the Gaussian elimination, and that Bill told the audience that Fujishige had obtained such a combinatorial one. I very much appreciated his remark, which made my result widely known to researchers who contributed thereafter to the great progress in algorithms for min-cost flows.

In the meantime, I announced in [39] that the approach shown there could be used to solve the submodular flow problem, while Tardos's seemed to be difficult to be applied to it. Before the publication of [39] Zimmermann wrote to me that he in collaboration with Hans Röck independently found the tree projection technique for min-cost flows and was investigating the submodular flow problem with the same approach. Since I had not then finished a paper on it, I proposed joint work with them. We carried out our joint work, communicating by air mail. I communicated only with Uwe Zimmermann, and I have not met Röck yet till now. This resulted in the joint paper [60], which shows a strongly polynomial algorithm for submodular flows with the oracle for SFM.

\section{Maximum flows}

In August, 2002 I was struggling with the research toward faster SFM algorithms. I once thought I had obtained a nice faster SFM algorithm but it turned out to be incorrect. I was disappointed but nevertheless tried to find how to remedy the error. Then I happened to find a new maximum flow algorithm for ordinary capacitated networks [42] (also see [43]). The algorithm is based on the Maximum-Adjacency ordering (MA ordering) for the capacitated directed networks and runs in weakly polynomial time. The complexity with scaling is the same as that of the existing best weakly polynomial one. Note that the MA ordering technique for undirected networks was then popular due to the celebrated paper of Hiroshi Nagamochi and Toshihide Ibaraki [95] (also see [109] and [96]). 
Since it did not improve the complexity of the existing algorithms, I hesitated to publish the algorithm but anyway submitted it to a journal ([42]). After some computational experiments made by Shigueo Isotani I presented a talk about it at the Oberwolfach meeting in November, 2002 (see [51]). Unexpectedly the talk drew participants' attention and brought intensive discussions in the evening on the behavior of the algorithm among the participants including Michel Goemans, Tom Liebling, Tom McCormick, Jens Vygen, and others.

As shown by Akiyoshi Shioura [107], the algorithm is not strongly polynomial. I have been wondering whether there is any way of modifying the algorithm so as to make it strongly polynomial (see, e.g., a variant [89] by means of preflow push). I have also been interested in the application of the MA-ordering max-flow technique to other combinatorial optimization problems.

\section{Generalized flows}

To find a strongly polynomial algorithm for min-cost generalized flows is a challenging open problem. It is a special case of the linear programming problem, so that it should be solved before the general LP would be. Even a further special case of finding a strongly polynomial algorithm for maximum generalized flows is still open.

I would like to see the behavior of the LP-Newton method for the generalized flow problems and its complexity.

\section{Trees, Arborescences, and Location Problems}

I enjoyed joint work with Makino and others at Osaka University, which has been continuing even after I moved to RIMS, Kyoto University in 2003 and he moved to the University of Tokyo in 2005.

One of the joint projects there was a problem of dynamically evacuating people through a tree network to multiple safety exits, and it motivated us to investigate the problem of locating multiple safety exits in dynamic tree networks (see, e.g., [87], [88]). We also investigated some other source location problems and related problems (see, e.g., [8], [102], [103]).

All through the above-mentioned joint projects with Makino and others I acted as an adviser and a discusser, and enjoyed the collaboration with them. The joint projects made me take more interest in trees, arborescences, and location problems.

In 2007 I was surprised to find a new theorem on disjoint arborescences shown by Naoyuki Kamiyama, Naoki Katoh, and Atsushi Takizawa at a domestic conference (see [82] for the theorem). The theorem generalizes the distinguished Edmonds' theorem on disjoint spanning arborescences, which was also motivated by an evacuation problem. But I had not fully understood or appreciated the essence of their theorem until András Frank visited RIMS and gave a talk related to their theorem in June, 2008, and I again tried to fully understand their proof of the theorem. Then I realized that they used the concept of 'reachability' from roots in their proof in an essential way but the real essence is in the 'convexity' of the vertex set reached from each 
root by an arborescence. The use of convexity lead us to a nice theorem on disjoint arborescences that properly generalized their theorem (see [47]). Since then I have been enjoying joint work with Kamiyama and others on disjoint arborescences and related subjects (see, e.g., [9]).

\section{Games and Discrete Convex Analysis}

When I moved to Osaka University in 1997, I worked with Murota (then at RIMS) about the investigation of his discrete convex/concave functions [93] and revealed the equivalence of $\mathrm{L}^{\natural}$-convex functions and Favati and Tardella's submodular integrally convex functions [22] (see [57] and also [93], [94]). This was my first commitment to Murota's discrete convex/concave functions although submodular analysis laid a basis of his discrete convex analysis ([45, Chapters IV and VII]).

In 1998 Zaifu Yang brought me a working paper of Faruk Gul and Ennio Stacchetti ([70]) and proposed to me joint work on the related subject. We soon noticed the close relationship between the gross substitutes condition and $\mathbf{M}^{\natural}$-concavity of Murota's discrete concave function. Though it took some years, the result finally appeared as [65].

In the meantime, I have long been interested in game theory, especially the GaleShapley stable matching (marriage) problem. In 2001 I found an interesting paper of Tamás Fleiner [23] about a generalization of the Gale-Shapley matching problem on matroids. I thought his result could further be generalized to a problem described by discrete concave functions of Murota. This resulted in a technical paper [19], which formed part of the 2002 master's thesis of Akinobu Eguchi but was left unpublished partly because he got a job at a private company after finishing his master's degree.

When I moved to RIMS, Kyoto University in April, 2003, Akihisa Tamura was with RIMS. Then I proposed to him joint work on extending the unpublished work [19] to two-sided matching markets with discrete concave functions. The joint work resulted in papers of [20], [62], and [63]. I am happy to see that Tamura has still been active in research on this subject and related topics.

\section{Dual Greedy Algorithms}

Dual greediness of a certain class of combinatorial optimization problems has been investigated in the literature. Among them is Frank's paper [25], which motivated my work of [44].

Gleb Koshevoy proposed joint work, related to [44], on generalizing matroids to those defined on convex geometries early in 2005 when he visited RIMS. We did not make any substantial progress and he went back to Moscow, leaving some possible axioms. In the summer of 2005, after proposing to Gleb to include Yoshio Sano, then a master's course student, as a collaborator of the joint work, Sano and I started to examine Gleb's axioms and found a critical error. We soon succeeded in rectifying 
the error to get a correct axiom to define a matroid on a convex geometry, called a cg-matroid. This resulted in [55], which forms the 2006 master's thesis of Sano.

Recently, I had a chance to make joint work with Ulrich Faigle on a further extension of dual greedy systems [21]. Also Hiroshi Hirai [72] made clear the detailed structure of dual greedy systems by means of greedy fans.

I would like to fully understand the structures of existing dual greedy systems, which could lead us to an extension of the intersection theorem for polymatroids and submodular systems.

\section{Concluding Remarks and Acknowledgments}

It should be my great pleasure if the present article with very personal reminiscence could stimulate and motivate further research of young scholars in the field of combinatorial and discrete optimization.

There are many other (joint) works about which I could not mention in the present article. I very much appreciate all of the kind efforts of the collaborators for joint work and support. In particular, I would like to express my sincere gratitude to Professor Emeritus Masao Iri of University of Tokyo for his invaluable advice and comments that have been leading my research in the right direction since 1975. I am also very grateful to Professor Bernhard Korte for his invitation to his Institute in Bonn for more than two years in total besides other short visits; especially the first one-year visit from October 1982 as an Alexander-von-Humboldt fellow was so exciting, stimulating, and enjoyable that I could meet a lot of great scholars of the staff and visitors there. I also greatly appreciate editorial efforts of Kazuo Murota for the present special issue and inviting me to write this article.

I have been enjoying research in combinatorial and discrete optimization till now and would also like to be enjoying it as long as I have any urge to do so.

\section{References}

1. A. I. Ali and H.-S. Han: Computational implementation of Fujishige's graph realizability algorithm. European Journal of Operational Research 108 (1998) 452-463.

2. K. Ando and S. Fujishige: On structures of bisubmodular polyhedra. Mathematical Programming 74 (1996) 293-317.

3. K. Ando, S. Fujishige and T. Naitoh: A greedy algorithm for solving a separable convex optimization problem on an integral bisubmodular polyhedron. Journal of the Operations Research Society of Japan 37 (1994) 188-196.

4. K. Ando, S. Fujishige and T. Naitoh: A greedy algorithm for minimizing a separable convex function over a finite jump system. Journal of the Operations Research Society of Japan 38 (1995) 362-375.

5. K. Ando, S. Fujishige and T. Naitoh: A characterization of bisubmodular functions. Discrete Mathematics 148 (1996) 299-303.

6. K. Ando, S. Fujishige and T. Nemoto: Decomposition of a signed graph into strongly connected components and its signed poset structure. Discrete Applied Mathematics 68 (1996) 237-248.

7. K. Ando, S. Fujishige and T. Nemoto: The minimum-weight ideal problem for signed posets. Journal of the Operations Research Society of Japan 39 (1996) 558-565.

8. K. Arata, S. Iwata, K. Makino and S. Fujishige: Locating sources to meet flow demands in undirected networks. Journal of Algorithms 42 (2002) 54-68. 
9. K. Bérczi, S. Fujishige, and N. Kamiyama: A linear-time algorithm to find a pair of arc-disjoint spanning in-arborescence and out-arborescence in a directed acyclic graph. Information Processing Letters 109 (2009) 1227-1231.

10. R. E. Bixby and W. H. Cunningham: Converting linear programs to network problems. Mathematics of Operations Research 5 (1980) 321-357.

11. R. Bixby, W. H. Cunnigham, and Topkis: Partial order of a polymatroid extreme point. Mathematics of Operations Research 10 (1985) 367-378.

12. R. E. Bixby and D. K. Wagner: An almost linear-time algorithm for graph realization. Mathematics of Operations Research 13 (1988) 99-123.

13. T. M. Cover: A proof of the data compression theorem of Slepian and Wolf for ergodic sources. IEEE Transactions on Information Theory IT-21 (1975) 226-228.

14. W. H. Cunningham: Testing membership in matroid polyhedra. Journal of Combinatorial Theory, Ser. B 36 (1984) 161-188.

15. W.H. Cunningham: On submodular function minimization. Combinatorica 5 (1985) 185-192.

16. W. H. Cunningham and A. Frank: A primal-dual algorithm for submodular flows. Mathematics of Operations Research 10 (1985) 251-262.

17. J. Edmonds: Submodular functions, matroids, and certain polyhedra. Proceedings of the Calgary International Conference on Combinatorial Structures and Their Applications (R. Guy, H. Hanani, N. Sauer and J. Schönheim, eds., Gordon and Breach, New York, 1970), pp. 69-87; also in: Combinatorial Optimization-Eureka, You Shrink! (M. Jünger, G. Reinelt and G. Rinaldi, eds., Lecture Notes in Computer Science 2570, Springer, Berlin, 2003), pp. 11-26.

18. J. Edmonds and R. Giles: A min-max relation for submodular functions on graphs. Annals of Discrete Mathematics 1 (1977) 185-204.

19. A. Eguchi and S. Fujishige, An extension of the Gale-Shapley matching algorithm to a pair of $\mathbf{M}^{\natural}$ concave functions, Discrete Mathematics and Systems Science Research Report 02-05, Osaka University, (2002).

20. A. Eguchi, S. Fujishige and A. Tamura: A generalized Gale-Shapley algorithm for a discrete-concave stable-marriage model. ISAAC 2003, LNCS 2906 (T. Ibaraki, N. Katoh, and H. Ono, Eds.), pp. 495504.

21. U. Faigle and S. Fujishige: A general model for matroids and the greedy algorithm. Mathematical Programming, Ser. A, 119 (2009) 353-369.

22. P. Favati and F. Tardella: Convexity in nonlinear integer programming. Ricerca Operativa 53 (1990) 3-44.

23. T. Fleiner: A matroid generalization of the stable matching polytope, in: B. Gerards and K. Aardal, eds., Proceedings of the 8th International IPCO Conference, LNCS 2081 (Springer-Verlag, Berlin, 2001) 105-114.

24. A. Frank: An algorithm for submodular functions on graphs. Annals of Discrete Mathematics 16 (1982) 189-212.

25. A. Frank: Increasing the rooted-connectivity of a digraph by one. Mathematical Programming 84 (1999) 565-576.

26. S. Fujishige: A primal approach to the independent assignment problem. Journal of the Operations Research Society of Japan 20 (1977) 1-15.

27. S. Fujishige: An algorithm for finding an optimal independent linkage. Journal of the Operations Research Society of Japan 20 (1977) 159-75.

28. S. Fujishige: Algorithms for solving the independent-flow problems. Journal of the Operations Research Society of Japan 21 (1978) 189-204.

29. S. Fujishige: Polymatroidal dependence structure of a set of random variables. Information and Control 39 (1978) 55-72.

30. S. Fujishige: "Independent flow" problems and submodular functions. Journal of the Faculty of Engineering, University of Tokyo A, No. 16 (1978) 42-43 (in Japanese)

31. S. Fujishige: Lexicographically optimal base of a polymatroid with respect to a weight vector. Mathematics of Operations Research 5 (1980) 186-196.

32. S. Fujishige: An efficient PQ-graph algorithm for solving the graph-realization problem. Journal of Computer and System Sciences 21 (1980) 63-86.

33. S. Fujishige: A note on the problem of updating shortest paths. Networks 11 (1981) 317-319.

34. S. Fujishige: Structures of polyhedra determined by submodular functions on crossing families. Mathematical Programming 29 (1984) 125-141. 
35. S. Fujishige: Submodular systems and related topics. Mathematical Programming Study 22 (1984) 113-131.

36. S. Fujishige: Theory of submodular programs—a Fenchel-type min-max theorem and subgradients of submodular functions. Mathematical Programming 29 (1984) 142-155.

37. S. Fujishige: On the subdifferential of a submodular function. Mathematical Programming 29 (1984) 348-360.

38. S. Fujishige: Combinatorial optimization problems described by submodular functions. 10th IFORS Conference, Washington D. C., 6-10 August, 1984. Operations Research '84 (J. P. Brans, ed., Elsevier Science Publishers B. V., 1984), pp. 379-392.

39. S. Fujishige: A capacity-rounding algorithm for the minimum cost circulation problem-a dual framework of the Tardos algorithm. Mathematical Programming 35 (1986) 298-308.

40. S. Fujishige: From classic flow problems to "neoflow" problems. Transactions of the Electronics, Information and Communication Engineers of Japan J70-A, No. 2 (1987) 3-16 (in Japanese).

41. S. Fujishige: A min-max theorem for bisubmodular polyhedra. SIAM Journal on Discrete Mathematics 10 (1997) 294-308.

42. S. Fujishige: A maximum flow algorithm using MA ordering. Operations Research Letters 31 (2003) 176-178.

43. S. Fujishige: Submodular function minimization and related topics. Optimization Methods and Software 18 (2003) 169-180.

44. S. Fujishige: Dual greedy polyhedra, choice functions, and abstract convex geometries. Discrete Optimization 1 (2004) 41-49.

45. S. Fujishige: Submodular Functions and Optimization (Annals of Discrete Mathematics 47) (NorthHolland, 1991); Second Edition (Annals of Discrete Mathematics 58) (Elsevier, 2005).

46. S. Fujishige: Theory of principal partitions revisited. In: W. Cook, L. Lovász, and J. Vygen (Editors): Research Trends in Combinatorial Optimization (Springer, Berlin, 2009), pp. 127-162.

47. S. Fujishige: A note on disjoint arborescences. Combinatorica 30 (2010) 247-252.

48. S. Fujishige, T. Hayashi, and K. Nagano: Minimizing continuous extensions of discrete convex functions with linear inequality constraints. SIAM Journal on Optimization 20 (2009) 856-867.

49. S. Fujishige, T. Hayashi, and S. Isotani: The minimum-norm-point algorithm applied to submodular function minimization and linear programming. Preprint No. RIMS-1517, RIMS, Kyoto University, September, 2006.

50. S. Fujishige, T. Hayashi, K. Yamashita, and U. Zimmermann: Zonotopes and the LP-Newton method. Optimization and Engineering 10 (2009) 193-205.

51. S. Fujishige and S. Isotani: New maximum flow algorithms by MA orderings and scaling. Journal of the Operations Research Society of Japan 46 (2003) 243-250.

52. S. Fujishige and S. Isotani: A submodular function minimization algorithm based on the minimumnorm base. Pacific Journal of Optimization 7 (2011) 3-17.

53. S. Fujishige and S. Iwata: Minimizing a submodular function arising from a concave function. Discrete Applied Mathematics 92 (1999) 211-215.

54. S. Fujishige and S. Iwata: Bisubmodular function minimization. SIAM Journal on Discrete Mathematics 19 (2006) 1065-1073.

55. S. Fujishige, G. A. Koshevoy, and Y. Sano: Matroids on convex geometries. Discrete Mathematics 307 (2007) 1936-1950.

56. S. Fujishige, X. Liu and X. Zhang: An algorithm for solving the minimum-norm point problem over the intersection of a polytope and an affine set. Journal of Optimization Theory and Applications 105 (2000) 113-141.

57. S. Fujishige and K. Murota: Notes on L-/M-convex functions and the separation theorems. Mathematical Programming, Ser. A 88 (2000) 129-146.

58. S. Fujishige and K. Nagano: A structure theory for the parametric submodular intersection problem. Mathematics of Operations Research 34 (2009) 513-521.

59. S. Fujishige and S. B. Patkar: The orthant non-interaction theorem for certain combinatorial polyhedra and its implications in the intersection and the Dilworth truncation of bisubmodular functions. Optimization 34 (1995) 329-339.

60. S. Fujishige, H. Röck and U. Zimmermann: A strongly polynomial algorithm for minimum cost submodular flow problems. Mathematics of Operations Research 14 (1989) 60-69. 
61. S. Fujishige, H. Sato and P. Zhan: An algorithm for finding the minimum-norm point in the intersection of a polyhedron and a hyperplane. Japan Journal of Industrial and Applied Mathematics 11 (1994) 245-264.

62. S. Fujishige and A. Tamura: A general two-sided matching market with discrete concave utility functions. Discrete Applied Mathematics 154 (2006) 950-970.

63. S. Fujishige and A. Tamura: A two-sided discrete-concave market with possibly bounded side payments: an approach by discrete convex analysis. Mathematics of Operations Research 32 (2007) 136155.

64. S. Fujishige and N. Tomizawa: A note on submodular functions on distributive lattices. Journal of the Operations Research Society of Japan 26 (1983) 309-318.

65. S. Fujishige and Z. Yang: A note on Kelso and Crawford's gross substitutes condition. Mathematics of Operations Research 28 (2003) 463-469.

66. S. Fujishige and P. Zhan: A dual algorithm for finding the minimum-norm point in a polytope. Journal of the Operations Research Society of Japan 33 (1990) 188-195.

67. S. Fujishige and P. Zhan: A dual algorithm for finding a nearest pair of points in two polytopes. Journal of the Operations Research Society of Japan 35 (1992) 353-365.

68. S. Fujishige and X. Zhang: New algorithms for the intersection problem of submodular systems. Japan Journal of Industrial and Applied Mathematics 9 (1992) 369-382.

69. G. Gallo, M. D. Grigoriadis and R. E. Tarjan: A fast parametric maximum flow algorithm and applications. SIAM Journal on Computing 18 (1989) 30-55.

70. F. Gul and E. Stacchetti: Walrasian equilibrium with gross substitutes. Journal of Economic Theory 87 (1999) 95-124.

71. T. S. Han: Nonnegative entropy measures of multivariate symmetric correlations. Information and Control 36 (1978) 133-156.

72. H. Hirai: Greedy fans: a geometric approach to dual greedy algorithms. Preprint No. RIMS-1508, RIMS, Kyoto University, June, 2005.

73. M. Iri: A necessary and sufficient condition for a matrix to be the loop or cut-set matrix of a graph and a practical method for the topological synthesis of networks. RAAG Research Notes, Ser. 3 No. 50 (1962); On the synthesis of loop and cutset matrices and the related problems. RAAG Memoirs A5 (1968) 4-38.

74. M. Iri: A practical algorithm for the Menger-type generalization of the independent assignment problem. Mathematical Programming Study 8 (1978) 88-105.

75. M. Iri: A review of recent work in Japan on principal partitions of matroids and their applications. Annals of the New York Academy of Sciences 319 (1979) 306-319.

76. M. Iri and N. Tomizawa: An algorithm for finding an optimal "independent assignment". Journal of the Operations Research Society of Japan 19 (1976) 32-57.

77. M. Iri, N. Tomizawa, and S. Fujishige: On the controllability and observability of a linear system with combinatorial constraints. Transactions of the Institute of Instrument and Control Engineers 13 (1977) 235-242 (in Japanese).

78. S. Iwata: A capacity scaling algorithm for convex cost submodular flows. Mathematical Programming, Ser. A 76 (1997) 299-308.

79. S. Iwata: Submodular function minimization. Mathematical Programming 112 (2008) $45-64$

80. S. Iwata, L. Fleischer and S. Fujishige: A combinatorial strongly polynomial algorithm for minimizing submodular functions. Journal of ACM 48 (2001) 761-777; also see: A combinatorial, strongly polynomial-time algorithm for minimizing submodular functions. Proceedings of the 32nd Annual ACM Symposium on Theory of Computing (Portland, OR, 2000), pp. 96-107.

81. S. Iwata and J. B. Orlin: A simple combinatorial algorithm for submodular function minimization. Proceedings of the twentieth Annual ACM-SIAM Symposium on Discrete Algorithms (2009), pp. 1230-1237.

82. N. Kamiyama, N. Katoh, and A. Takizawa: Arc-disjoint in-trees in directed graphs. Combinatorica 29 (2009) 197-214.

83. G. Kishi and Y. Kajitani: On maximally distant trees. Proceedings of the 5th Annual Allerton Conference on Circuit and System Theory (1967), pp. 635-643; Maximally distant trees and principal partition of a linear graph. IEEE Transactions on Circuit Theory CT-16 (1969) 323-330.

84. E. L. Lawler: Matroid intersection algorithms. Mathematical Programming 9 (1975) 31-56.

85. E. L. Lawler and C. U. Martel: Computing maximal polymatroidal network flows. Mathematics of Operations Research 7 (1982) 334-347. 
86. L. Lovász: Submodular functions and convexity. In: Mathematical Programming-The State of the Art (A. Bachem, M. Grötschel and B. Korte, eds., Springer, Berlin, 1983), pp. 235-257.

87. S. Mamada, K. Makino and S. Fujishige: Optimal sink location problem for dynamic flows in a tree network. IEICE Transactions on Fundamentals E85-A (2002) 1020-1025.

88. S. Mamada, T. Uno, K. Makino and S. Fujishige: $\mathrm{An} \mathrm{O}\left(n \log ^{2} n\right)$ algorithm for the optimal sink location problem on dynamic tree networks. Discrete Applied Mathematics 154 (2006) 2387-2401.

89. Y. Matsuoka and S. Fujishige: Practical efficiency of maximum flow algorithms using MA orderings and preflows. Journal of the Operations Research Society of Japan 48 (2005) 297-307.

90. S. T. McCormick and S. Fujishige: Strongly polynomial and fully combinatorial algorithms for bisubmodular function minimization. Mathematical Programming, Ser. A 122 (2010) 87-120.

91. C. J. H. McDiarmid: Rado's theorem for polymatroids. Mathematical Proceedings of the Cambridge Philosophical Society 78 (1975) 263-281.

92. N. Megiddo: Optimal flows in networks with multiple sources and sinks. Mathematical Programming 7 (1974) 97-107.

93. K. Murota: Discrete convex analysis. Math. Programming 83 (1998) 313-371.

94. K. Murota: Discrete Convex Analysis (SIAM Monographs on Discrete Mathematics and Applications 10, SIAM, 2003).

95. H. Nagamochi and T. Ibaraki: Computing edge-connectivity in multigraphs and capacitated graphs SIAM Journal on Discrete Mathematics 5 (1992) 54-66.

96. H. Nagamochi and T. Ibaraki: Graph connectivity and its augmentation: applications of MA orderings. Discrete Applied Mathematics 123 (2002) 447-472.

97. H. Narayanan: Theory of Matroids and Network Analysis, Ph.D Thesis, Department of Electrical Engineering, Indian Institute of Technology, Bombay, February 1974

98. T. Ohto: An experimental analysis of the Bixby-Wagner algorithm for graph realization problems (in Japanese). Research Report of the Special Interest Group for Algorithms of the Information Processing Society of Japan, Algorithms 84-1 (2002) 1-8.

99. J. B. Orlin: A faster strongly polynomial algorithm for submodular function minimization. Mathematical Programming, Ser. A 118 (2009) 237-251.

100. A. Recski: Matroid Theory and its Applications in Electric Network Theory and in Statics (Algorithms and Combinatorics 6, Springer, Berlin, 1989).

101. R. T. Rockafellar: Convex Analysis (Princeton University Press, Princeton, N.J., 1970).

102. M. Sakashita, K. Makino, and S. Fujishige: Minimum cost source location problems with flow requirements. Algorithmica 50 (2008) 555-583.

103. M. Sakashita, K. Makino, H. Nagamochi, and S. Fujishige: Minimum transversals in posi-modular systems. SIAM Journal on Discrete Mathematics 23 (2009) 858-871.

104. P. Schönsleben: Ganzzahlige Polymatroid-Intresektions-Algorithmen, Dissertation, Eidgenössische Technische Hochschule Zürich, 1980.

105. A. Schrijver: A combinatorial algorithm minimizing submodular functions in strongly polynomial time. Journal of Combinatorial Theory, Ser. B 80 (2000) 346-355.

106. L. S. Shapley: Cores of convex games. International Journal of Game Theory 1 (1971) 11-26.

107. A. Shioura: The MA-ordering max-flow algorithm is not strongly polynomial for directed networks. Operations Research Letters 32 (2004) 31-35.

108. D. Slepian and J. Wolf: Noiseless coding of correlated information sources. IEEE Transactions on Information Theory IT-19 (1973) 471-480.

109. M. Stoer and F. Wagner: A simple min cut algorithm. Journal of ACM 44 (1997) 585-591.

110. É. Tardos: A strongly polynomial minimum cost circulation algorithm. Combinatorica 5 (1985) 247-256.

111. N. Tomizawa: Strongly irreducible matroids and principal partition of a matroid into strongly irreducible minors (in Japanese). Transactions of the Institute of Electronics and Communication Engineers of Japan 59A (1976) 83-91.

112. N. Tomizawa: An $\mathrm{O}\left(\mathrm{m}^{3}\right)$ algorithm for solving the realization problem of graphs-On combinatorial characterizations of graphic $(0,1)$-matrices (in Japanese). Papers of the Technical Group on Circuits and System Theory, Institute of Electronics and Communication Engineers of Japan, CST75106 (1976).

113. K. Truemper: Matroid Decomposition (Academic Press, Boston, 1992).

114. W. T. Tutte: An algorithm for determining whether a given binary matroid is graphic. Proceedings of the American Mathematical Society 11 (1960) 905-917. 
115. W. T. Tutte: Connectivity in Graphs (University of Toronto Press, Toronto, 1966).

116. P. Wolfe: Finding the nearest point in a polytope. Mathematical Programming 11 (1976) 128-149

117. U. Zimmermann: Minimization on submodular flows. Discrete Applied Mathematics 4 (1982) 303 323 